\title{
Housing for health promotion
}

\author{
Roderick J. Lawrence
}

Published online: 11 October 2011

(C) Swiss School of Public Health 2011

\section{Introduction}

The relation between the residential environment and health is multidimensional and complex. It is possible not only to determine whether housing promotes or hinders health and quality of life, but also how the health of an individual can influence her/his housing conditions. Housing conditions, and homelessness in particular, are key components in the chain of explanatory factors linking poverty and inequality to health status.

Some empirical research has identified and measured the mechanisms linking characteristics of residential environments to mental health. Studies like those included in this issue confirm that people living in housing units that are in a poor state that lack natural daylight and ventilation, or that is prone to vandalism, have a relatively low mental health status.

\section{Synopsis of research findings}

Housing is an important determinant of quality of life. It can influence health promotion. The multiple components of housing units and their surroundings need to be considered in terms of their potential and effective contribution to the physical, social and mental well-being. In principle,

This paper belongs to the special issue "Housing for health promotion".

R. J. Lawrence $(\square)$

Institute of Environmental Sciences, Human Ecology Group,

University of Geneva, Battelle, Building D, 7 route de Drize,

1227 Geneva, Carouge, Switzerland

e-mail: roderick.lawrence@unige.ch

there are nine main dimensions of residential environments that ought to be considered. That include:

1. The characteristics of the site, in ensuring safety from "natural" disasters including earthquakes, landslides, flooding and fires; and protection from any potential source of natural radon. In addition, the impacts of industrial accidents should be considered.

2. The residential building as a shelter for the inhabitants from the extremes of outdoor temperature; as a protector against dust, insects and rodents; as a provider of security from unwanted persons; and as an insulator against noise. Sleep disturbance is a risk factor of stress and related illnesses.

3. The effective provision of a safe and continuous supply of water that meets standards for human consumption, and the maintenance of sewage and solid waste disposal. Water can be a vector of infectious diseases and it is still a serious public health challenge in many countries.

4. Ambient atmospheric conditions in the residential neighbourhood and indoor air quality. Both are related to emissions from industrial production, transportation, fuels used for domestic cooking and heating, as well as the local climate and ventilation inside and around buildings. Some cooking and heating appliances emit fumes from fuels that have adverse effects on human respiratory systems.

5. Household occupancy conditions, which can influence the transmission of airborne infections including pneumonia and tuberculosis, and the incidence of injury from domestic accidents. Data show that domestic accidents are a major public health challenge.

6. Access to community facilities and services (for commerce, education, employment, leisure and 
primary health care) which are affordable and available to all individuals and groups. An increasing number of people in Australasia, Europe and North America rely on social welfare in order to access primary health care and affordable housing.

7. Food safety, including provision of uncontaminated fresh foods that can be stored with protection against spoilage. The type, amount and quality of food eaten have a direct impact on health and should be considered when considering the increasing incidence of obesity and diabetes type 2 in many countries.

8. The control of vectors and host of disease outdoors and inside residential buildings which can propagate in the building structure; the use of non-toxic materials and finishes for housing and building construction; the use and storage of hazardous substances or equipment in the residential environment.

9. The capacity of housing to sustain positive social and psychological processes. For example, the capacity of the resident to use her/his domestic space to control personal contacts with others, to create a personal space using physical and psychological appropriation practises.
Some contributions highlight the importance of domestic activities and the lifestyles of individuals and households. Studies in several countries show that more than half of all non-sleep activities of employed people between 18 and 64 years of age occur inside housing units. Children, the aged and housewives spend even more time indoors. Therefore, any shortcomings in the indoor residential environment, including high household population density, may have implications on human health and well-being.

Each of the articles in this special issue presents an original empirical study of the interrelations between the housing and health. The disciplinary diversity and the geographical distribution of the authors included in this issue confirm that there is a widely shared interest around the world for this complex subject. The Guest Editor of this special issue would like to thank the Scientific Committee of the 20th IUHPE World Conference on Health Promotion held in Geneva from 11 to 15 July 2010 for the opportunity to organize a sub-plenary session on 'Health promoting architecture' and a workshop on 'Health promoting strategies for housing and health'. Also thanks to each of the anonymous reviewers who have peer reviewed all the papers submitted for this issue. 